\title{
Propranolol inhibits stemness of hemangioma through Jagged1
}

\author{
Xiaorong $\mathrm{Ma}^{1}$, Kaiyang $\mathrm{Lv}^{1}$, Liming $\mathrm{Wu}^{2}$, Tianxiang Ouyang ${ }^{1}$ \\ ${ }^{1}$ Department of Plastic and Reconstructive Surgery, Xinhua Hospital, Shanghai Jiao Tong University School of Medicine, Shanghai, China; \\ ${ }^{2}$ Department of Endocrinology, Xuhui District Central Hospital, Shanghai, China \\ Contributions: (I) Conception and design: X Ma; (II) Administrative support: X Ma; (III) Provision of study materials or patients: X Ma; (IV) \\ Collection and assembly of data: All authors; (V) Data analysis and interpretation: X Ma; (VI) Manuscript writing: All authors; (VII) Final approval of \\ manuscript: All authors. \\ Correspondence to: Tianxiang Ouyang. Department of Plastic and Reconstructive Surgery, Xinhua Hospital, Shanghai Jiao Tong University School of \\ Medicine, 1665 Kongjiang Road, Shanghai 200092, China. Email: ouyangtianxiang@xinhuamed.com.cn; Liming Wu. Department of Endocrinology, \\ Xuhui District Central Hospital, 966 Middle Huaihai Road, Shanghai 200031, China. Email: wuliming_sh@hotmail.com.
}

\begin{abstract}
Background: Propranolol is used clinically to treat infantile hemangioma (IH), although the exact mechanism that underlies its effectiveness is not fully understood. The Jagged1/Notch signaling pathway is downstream of the $\beta 2$-adrenergic receptor ( $\beta 2$-AR). Propranolol is a non-selective $\beta 2$-AR blocker that was shown to inhibit demethylation adrenaline-induced Jagged1 expression. A previous study has shown that propranolol dose-dependently inhibits the growth of IH. However, the effects of propranolol on stemness of $\mathrm{IH}$ are not known and are thus addressed in the current study.
\end{abstract}

Methods: We analyzed the expression of Jagged 1 and Notch3 in IH specimens, using genetic tools to alter Notch signaling. The transduced IH cells were treated with different doses of propranolol, and the effects on IH cell proliferation, migration, and potential for tumor sphere formation were investigated. The effects of altered Notch signaling on tumor formation in vivo were also assessed.

Results: Notch3 and Jagged1 were significantly upregulated in IH. Augmented Notch signaling in IH cells increased cell proliferation, migration, the potential for tumor sphere formation and in vivo tumor formation. On the other hand, reduced Notch signaling in IH cells decreased cell proliferation, migration, the potential for tumor sphere formation and in vivo tumor formation.

Conclusions: Jagged1/Notch signaling regulated the stemness of IH, and propranolol inhibited it through suppression of Notch signaling.

Keywords: Cancer stem cells (CSCs); infantile hemangioma (IH); Jagged1/Notch; propranolol; stemness

Submitted Sep 22, 2021. Accepted for publication Nov 17, 2021.

doi: $10.21037 / \mathrm{atm}-21-5563$

View this article at: https://dx.doi.org/10.21037/atm-21-5563

\section{Introduction}

Infantile hemangioma (IH) is one of the commonest benign tumors in infants and young children, with a prevalence of $3-10 \%$ (1). Based on its unique developmental cycle, IH has two stages: proliferation and regression (2). Cancer stem cells (CSCs) persist in IH, with characteristic CD133 expression (3) as a signature. These CSCs have unlimited proliferation potential and are specifically resistant to radiotherapy and chemotherapy. Different soluble cytokines (including vascular endothelial growth factor (VEGF), insulin-like growth factors, etc.) can upregulate the expression of a variety of stem cell genes in IH, including Notch, fibroblast growth factors, Wnt, etc., to maintain the proliferation potential of the stem cells without initiating differentiation into a specific cell type (4).

The Jagged1/Notch signaling pathway plays an important role in stem cell commitment (5). The biological behavior of bone marrow hematopoietic stem cells (HSCs) is regulated through the Jagged1/Notch1 signaling pathway, which is crucial for HSCs to maintain both their proliferative potential and undifferentiated 
state (6). Specifically, cancer cells can secrete soluble Jagged1 molecules to activate the Notch signaling pathway to promote tumor growth (7-11). In many cancers the Jagged1/Notch signaling pathway regulates the tumor formation rate, metastatic ability and resistance to chemotherapeutics of cells in vivo (7-11). In some malignant tumors that are not sensitive to chemotherapeutic drugs, the targeted tumor has been shown to activate the Jagged1/ Notch signaling pathway to increase cancer cell stemness to resist the damage from chemotherapy (12-14).

The Jagged1/Notch signaling pathway is abnormally expressed in IH. In 2009, Calicchio et al. found through genomics research that in proliferative $\mathrm{IH}$, the expression of Notch 4 and Jagged 1 increased by 3.15- and 6.52-fold, respectively (15). In IH, cross talk between the VEGF and Notch pathways likely coordinates tumor progression (16). Notch 3 and Jagged 1 are expressed by IH (17), and further research by Boscolo et al. found that Jagged1 binds to the Notch3 receptor on the stem cell surface after cell contact, which activates the Notch3 receptor and downstream signals to initiate the differentiation of IH cells into pericytes (18). Blocking the binding of Jagged 1 to its receptor significantly reduced the differentiation efficiency (18). Together, these reports demonstrate the critical role of the Jagged1/Notch signaling pathway in the tumorigenesis of $\mathrm{IH}$.

The Jagged1/Notch signaling pathway is downstream of the $\beta 2$-adrenergic receptor ( $\beta 2$-AR) (19). Hence, $\beta 2$ AR blockers are expected to have a suppressive effect on Jagged1/Notch signaling and subsequent tumor stemness. Propranolol is a non-selective $\beta 2$-AR blocker that was shown to inhibit demethylation adrenaline-induced Jagged1 expression (20). A previous study has shown that propranolol dose-dependently inhibits the growth of IH (21). However, the effects of propranolol on stemness of $\mathrm{IH}$ are not known. Therefore, we hypothesized that propranolol may inhibit $\beta 2-A R$ and the downstream Jagged1/Notch signaling pathway to thus affect the stemness of IH. This hypothesis was tested in the current study. We present the following article in accordance with the ARRIVE reporting checklist (available at https://dx.doi.org/10.21037/atm-21-5563).

\section{Methods}

\section{Ethics approval and consent to participate}

Animal experiments were performed under a project license (No. XHEC-F-2021-072) granted by Ethics Committee of Shanghai Jiao Tong University School of Medicine. All animal experiments were carried out in compliance with the relevant guidelines and regulations for the care and use of animals issued by the Institutional Research Ethics Committee of Shanghai Jiao Tong University. A protocol was prepared before the study without registration.

All procedures performed in this study involving human participants were in accordance with the Declaration of Helsinki (as revised in 2013). The study was approved by Institutional Ethics Board of the Shanghai Jiao Tong University School of Medicine (No. XHEC-D-2021-161) and informed consent was taken from all the patients.

All clinical data were anonymized, and all identifiable information and biological samples were stored according to local guidelines. For each animal experiment, power calculations $(\mathrm{P}<0.05)$ were performed to include sufficient numbers of animals. An allocation concealment method was applied for randomization in the allocating of experimental units to control and treatment groups. No criteria were used for excluding animals (or experimental units) during the experiment, and no data were excluded during the analysis. The study did not have humane endpoints.

\section{Sample collection, cell isolation, culture and sorting}

A total of $5 \mathrm{IH}$ patients who had undergone surgical removal of IH tissue, but no preoperative medical treatment (including corticosteroids and propranolol), were recruited at the Xinhua Hospital of Shanghai Jiao Tong University. $\mathrm{IH}$ tissue and paired normal skin samples (NSk; from the same patient) were collected for analysis. Briefly, both samples were washed with phosphate-buffered saline (PBS) and minced to a fine paste followed by a PBS wash. The tissues were then digested with $2 \mathrm{mg} / \mathrm{mL}$ collagenase (Sigma-Aldrich, Rockville, MD, USA) for $\approx 2.5 \mathrm{~h}$ in a shaker at $37^{\circ} \mathrm{C}$. The dispersed cells were filtered through a $66-\mu \mathrm{mol} / \mathrm{L}$ nylon cell strainer, and then subjected to flow cytometry-based sorting for CD31-positive cells using a PE-cy7-conjugated mouse anti-human CD31 antibody (Becton-Dickinson Biosciences, San Jose, CA, USA). The obtained cells were cultured in Dulbecco's Modified Eagle Medium (DMEM, Gibco, Shanghai, China) supplemented with $5 \%$ fetal bovine serum (FBS; Sigma-Aldrich) in a $5 \%$ $\mathrm{CO}_{2}$ incubator set at $37^{\circ} \mathrm{C}$. Flow cytometry for isolating virally transduced cells was based on green fluorescent protein (GFP) direct fluorescence.

\section{Cell transduction}

The complete coding sequence for Jagged1 was obtained by 
PCR using cDNA from the IH tissue of a human patient as a template. The sequence for small hairpin interfering RNA (shRNA) that targets and inhibits the translation of human Jagged 1 mRNA (shJagged1) was 5'-AGATCACTGTTTA GATTTGCCATAGAG-3', as described (22). The control applied a scrambled sequence. The adeno-associated virus (AAV) carried either the Jagged1 transgene or shJagged1, or the scrambled sequence under the control of a ubiquitous cytomegalovirus (CMV) promotor. A luciferase and a GFP reporter were connected by a $\mathrm{p} 2 \mathrm{~A}$ to allow simultaneous expression. The GFP reporter made the transduced cells visible and sortable by flow cytometry, while the luciferase reporter allowed the transduced cells to be traceable in living mice. HEK293T cells were maintained in DMEM (Invitrogen, Rockville, MD, USA) suppled with 5\% fetal bovine serum (FBS; Sigma-Aldrich, Rockville, MD, USA) in a humidified incubator at $37^{\circ} \mathrm{C}$ with a $5 \% \mathrm{CO}_{2}$ atmosphere. Co-transfection of HEK293 T cells with prepared plasmids, serotype 2 helper plasmids and packaging plasmids was performed in a lipofectamine-3000 assay, followed by purification and titration. In vitro transduction of IH cells used a multiplicity of infection of 100 and the transduced cells were analyzed after $96 \mathrm{~h}$.

\section{Animal experiments}

To receive xenografts and for analysis of bioluminescence, 12 -week-old male nude mice (3 groups, 6 mice in each group, female and male mice evenly distributed to exclude a possibility of the effects of sex on the experimental results; SLAC Laboratory Animal Co., Ltd., Shanghai, China) were anesthetized with $2.5 \%$ isoflurane inhalation. There were no expected or unexpected adverse events. Transduced IH cells (200 cells/mouse) were subcutaneously grafted above the root of the tail and the xeno-tumor was assessed with a bioluminescent assay (IVIS imaging system, Perkin Elmer, Santa Clara, CA, USA) at 1 month after transplantation. Briefly, 10 min before IVIS imaging, mice received an intraperitoneal injection of $200 \mathrm{mg} / \mathrm{kg}$ luciferin (SigmaAldrich) were anesthetized with $2.5 \%$ inhaled isoflurane. The in vivo imaging system imaging parameters used standard protocols with optimization. Bioluminescence was determined as twice the background-subtracted ocular region of interest (ROI) flux from the 20-min imaging window. The bioluminescence level was obtained by subtracting the value at baseline from the value at the peak. The mice were humanely killed by $2.5 \%$ isoflurane inhalation followed by cervical dislocation. of nude mice.

\section{Tumor sphere formation}

The cells were cultured at a density of $1 \times 10^{4}$ cells/well in tumor sphere media (composed of DMEM, $20 \mathrm{ng} / \mathrm{mL}$ recombinant human epidermal growth factor, $20 \mathrm{ng} / \mathrm{mL}$ basic fibroblast growth factor, $10 \mathrm{ng} / \mathrm{mL}$ leukemia inhibitory factor and $60 \mu \mathrm{g} / \mathrm{mL} \mathrm{N}$-acetylcysteine; Gibco) and then exposed to different treatments to be analyzed after certain time intervals.

\section{Cell proliferation assay}

The proliferative potential of IH cells was assessed with an MTT assay (Roche, Indianapolis, IN, USA), in which the absorbance value (OD) of the cells was quantified at $540 \mathrm{~nm}$.

\section{Immunobistochemistry and western blot}

Immunohistochemistry and western blots were done as routine, using primary antibodies including rabbit antiJagged1, anti-Notch3, anti-NICD (Notch intracellular domain), anti-HES1 and anti- $\beta$-actin (ab7771, ab23426, ab8925, ab108937 and ab8227; Abcam, Cambridge, MA, USA). The secondary antibody used a horseradishperoxidase-conjugated anti-rabbit antibody (Jackson ImmunoResearch Labs, West Grove, PA, USA). The images were taken using microscopes (Nikon, Tokyo, Japan).

\section{Real-time quantitative PCR (RT-qPCR)}

A SYBR Green PCR Kit (Qiagen, Shanghai, China) was used for RT-qPCR with the designed primers purchased from Qiagen. The primers were: Notch3/NICD: 5'-GTGTGTGTCAATGGCTGGAC-3' (forward) and 5'-GTGACACAGGAGGCCAGTCT-3' (reverse); Jagged 1: 5'-ATCGTGCTGCCTTTCAGTTT-3' (forward) and 5'-GGTCACGCGGATCTGATACT-3' (reverse); $\beta$-actin: 5'-CTCTTCCAGCCTTCCTTCCT-3' (forward) and 5'-AGCACTGTGTTGGCGTACAG-3' (reverse). The RT-qPCR reactions were performed in triplicate. The gene values were assessed with the $2^{-\Delta \Delta C t}$ method and obtained after sequential normalization to $\beta$-actin and the experimental controls.

\section{Cell migration assay}

Differently treated cells were seeded in the top chamber of a transwell insert (Millipore, Bedford, MA, USA) in serum- 

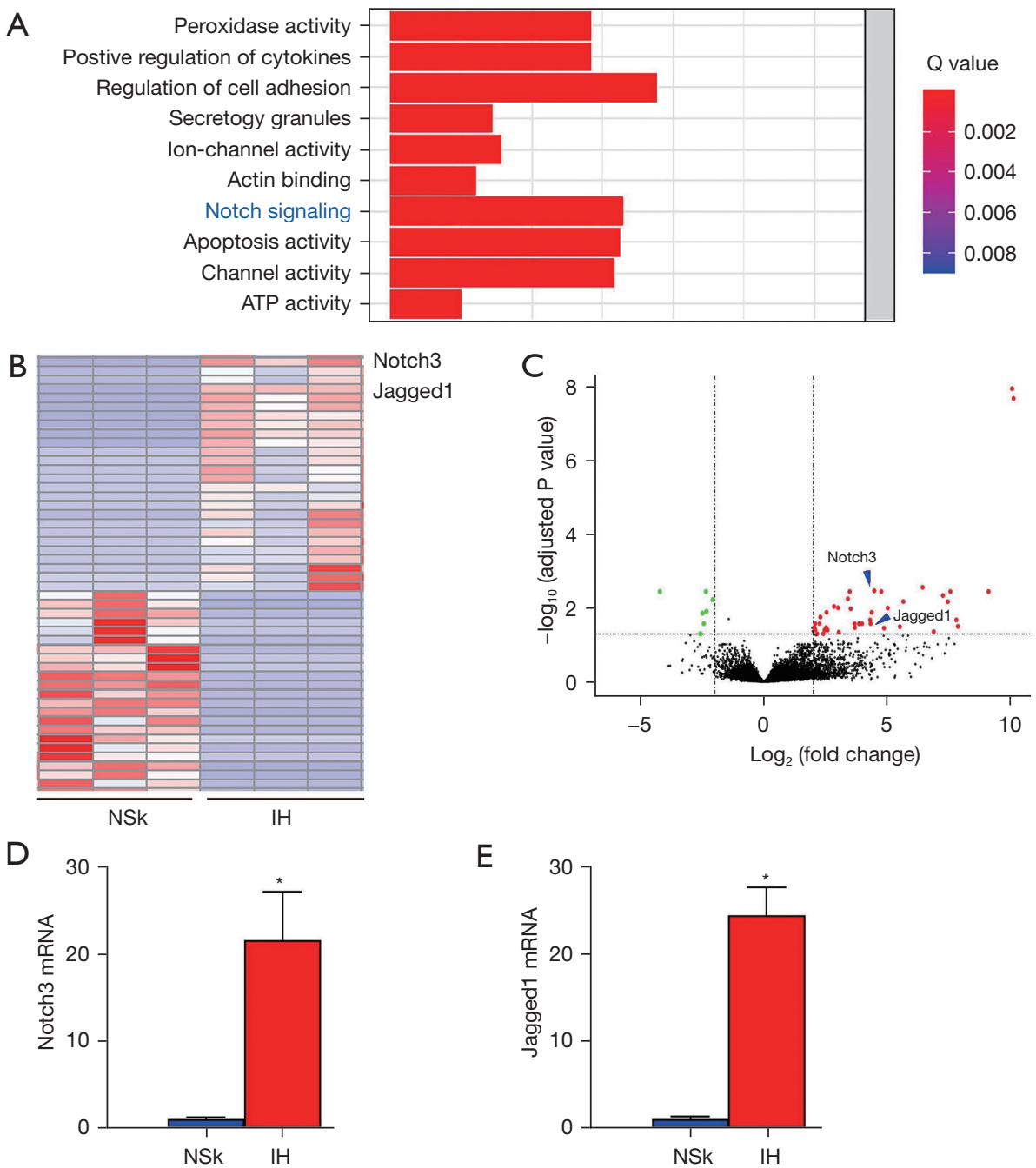

Figure 1 Bioinformatic analysis of the activation of Notch signaling in infantile hemangioma (IH) using human hemangioma data (GSE100682). The gene filing in the IH samples was compared with that of paired normal skin samples (NSk). (A) Bar plot shows that Notch signaling was significantly enhanced in IH, compared with NSk. Differentiation analysis shows that Notch3 and Jagged1 from the Notch signaling pathway were both expressed at significantly higher levels in IH compared with NSk, presented as a heat map (B) and a volcano map (C). Fold increases in Notch3 (D) and Jagged1 (E). ${ }^{*} \mathrm{P}<0.05$.

free medium, while the lower chamber of the transwell was filled with medium supplemented with $10 \%$ FBS. The cells that migrated to the lower surface were fixed with methanol, stained with crystal violet, and then quantified in $48 \mathrm{~h}$.

\section{Statistical analysis}

The data was summarized and statistically analyzed with GraphPad Prism 7 (GraphPad, Chicago, IL, USA). One-way ANOVA was performed to compare the data from different groups. The values are expressed as mean \pm standard deviation. Significance was considered when $\mathrm{P}<0.05$.

\section{Results}

\section{Bioinformatic analysis of activation of Notch signaling in IH}

In order to under the molecular mechanisms that underlie the development of $\mathrm{IH}$, we performed data mining of a published database, but to our surprise, the available databases for $\mathrm{IH}$ are limited. However, we did find a GEO source of human hemangioma data (GSE100682) that allowed us to perform an analysis of the gene filing in the IH samples, compared with the paired (NSk) samples. Among all the signaling pathways that were significantly upregulated in $\mathrm{IH}$, we found that Notch signaling was pronounced, as shown in Figure $1 \mathrm{~A}$. 
A

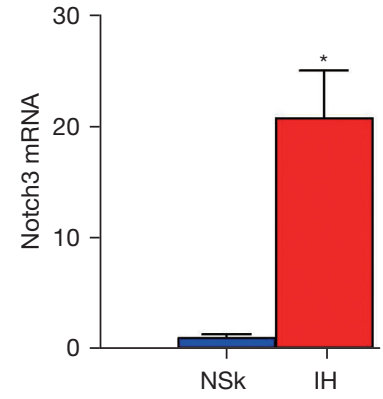

C

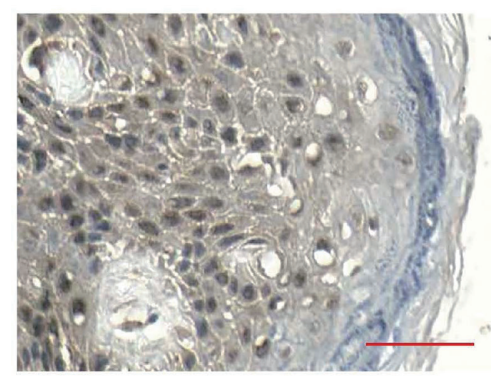

NSk

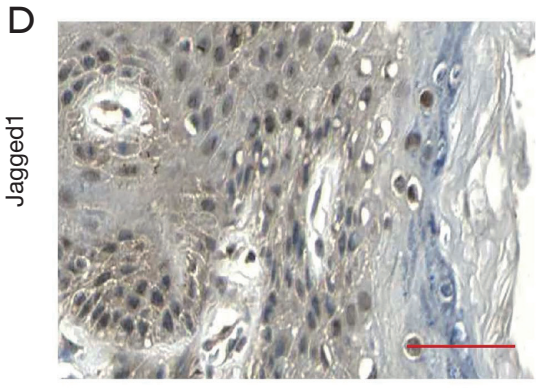

NSk
B
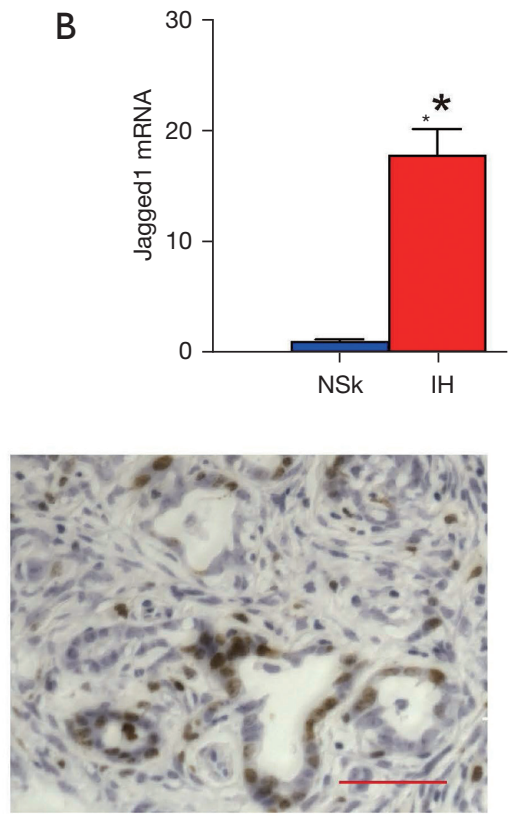

$\mathrm{IH}$

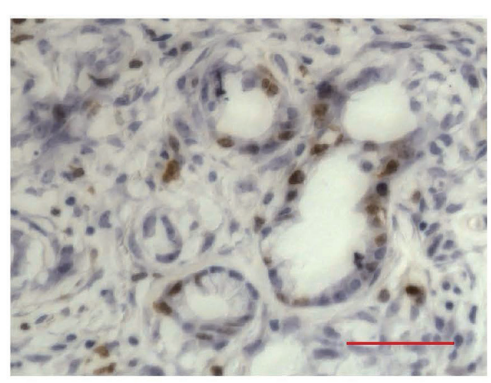

$\mathrm{IH}$

Figure 2 Analysis of 5 human infantile hemangioma (IH) specimens with the paired NSk for activation of Notch signaling. RT-qPCR for Notch3 (A) and Jagged1 (B) in the IH and paired NSk samples. Representative immunostaining of Notch3 (C) and Jagged1 (D) in the IH and paired NSk samples. $n=5 .{ }^{*} \mathrm{P}<0.05$. Scale bars $=50 \mu \mathrm{m}$.

The Limma R package identified Notch3 and Jagged1 from the Notch signaling pathway, which were all expressed at significantly higher levels in IH compared with NSk, as shown in a heat map (Figure 1B) and a volcano map (Figure 1C). The fold increases are shown for Notch3 (Figure 1D) and Jagged 1 (Figure 1E). Together, the bioinformatic analyses suggested that Notch signaling is activated in IH.

\section{Activation of Notch signaling in IH specimens}

Next, we analyzed the $5 \mathrm{IH}$ specimens from the patients and the paired NSk were used as controls (Table S1). We detected significantly higher expression of Notch3 (Figure $2 A$ ) and Jagged1 (Figure 2B) in the IH specimens.
The fold increase of both factors was similar to those reported by GSE100682 (Figures $1 D, 1 E, 2 A, 2 B$ ). Next, immunostaining was performed in the IH and paired NSk samples. Although Notch3 (Figure 2C) and Jagged1 (Figure 2D) were hardly detected in the NSk samples, strong nuclear staining of Notch3 (Figure 2C) and Jagged1 (Figure 2D) was detected in the IH samples, confirming the findings from the mRNA analysis. Together, the findings suggested that Notch signaling is potently activated in IH.

\section{Genetic alteration of activation levels of Notch signaling in IH cells}

In order to study the role of Notch signaling in IH, 


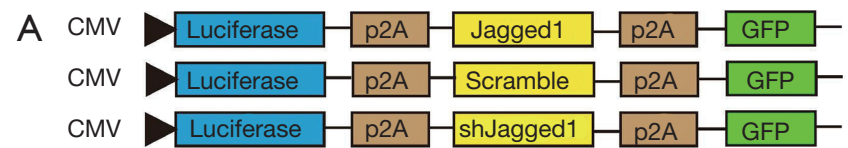

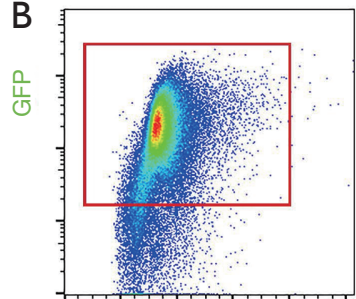

$\mathrm{IH}$-shJagged 1

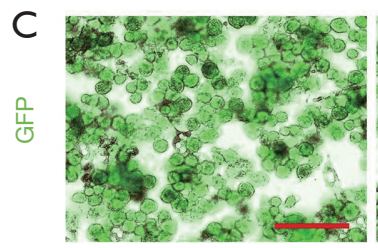

$\mathrm{IH}$-shJagged 1

D

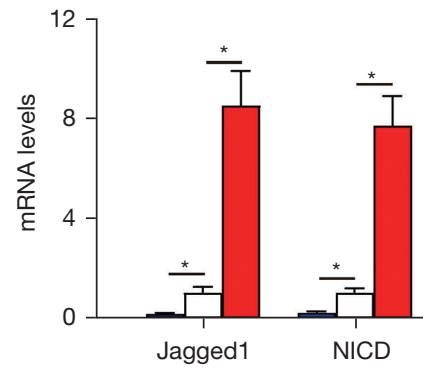

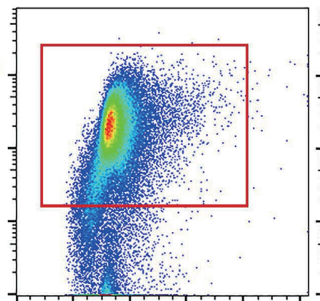

$\mathrm{IH}$-scramble

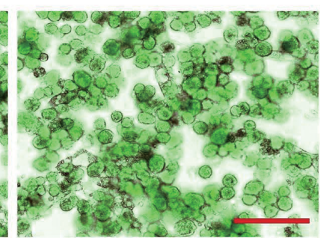

IH-scramble

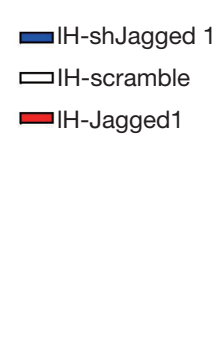

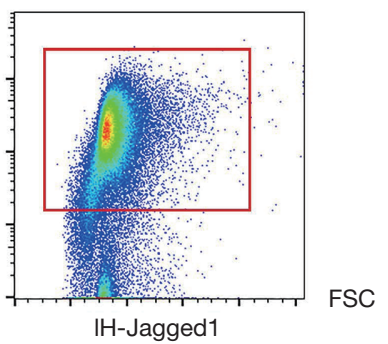

IH-Jagged 1

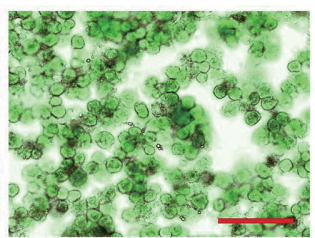

IH-Jagged1

$\mathrm{E}$

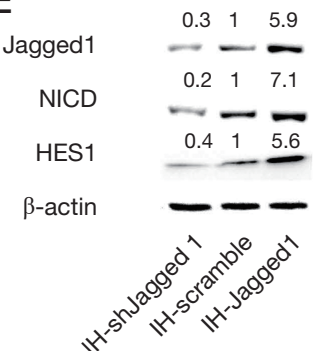

Figure 3 Genetic alteration of activation levels of Notch signaling in infantile hemangioma (IH) cells. (A) Schematic of adeno-associated virus (AAV) that carried either Jagged1, shJagged1 or control scrambled sequence, under the control of a ubiquitous cytomegalovirus promotor. A luciferase reporter and a green fluorescent protein (GFP) reporter were co-expressed by connection to the transgenics with p2A sequences. The transduced cells were fluoresced green due to GFP. (B) Representative flow charts for transduced IH cells with different AAVs. (C) Cell in culture. (D) RT-qPCR for Jagged1 and NICD in transduced cells. (E) Western blot for Jagged1, NICD and HES1. n=5. ${ }^{*} \mathrm{P}<0.05$. Scale bars $=50 \mu \mathrm{m}$. Notch intracellular domain. Hes1, hairy and enhancer of split-1.

we designed an AAV-mediated genetic manipulation strategy to allow either activation of Notch signaling by overexpressing Jagged1, or deactivation of Notch signaling by depleting Jagged 1 through the expression of shRNA for Jagged1 (shJagged1), both under the control of a ubiquitous CMV promotor. A luciferase and a GFP reporter are both co-expressed, which was fulfilled through connecting $\mathrm{p} 2 \mathrm{~A}$ sequences among the transgenes (Figure $3 A$ ). A scrambled sequence was used as a control for Jagged 1 and shJagged 1 (Figure $3 A$ ). The GFP made the cells fluoresce green as a label of the transgene, Jagged1, scrambled, or shJagged 1 . The luciferase reporter allowed us to trace these cells in the living mice. Transduction of a selected primary IH line obtained from the 5 human IH specimens with these AAVs reached a very high infection efficiency, as shown by the flow cytometry analysis based on GFP (Figure 3B). We then examined the levels of Jagged 1 and a Notch factor downstream of the connection between Notch3 and Jagged1, NICD, in these transduced cells. We found the overexpression of Jagged 1 significantly increased the levels of Jagged1 and NICD mRNA, while depletion of Jagged1 significantly decreased their levels, in IH cells (Figure 3C). Moreover, the overexpression of Jagged 1 significantly increased the protein levels of Jagged1, NICD and a NICD-downstream factor, Hairy and enhancer of split-1 (HES1), while depletion of 


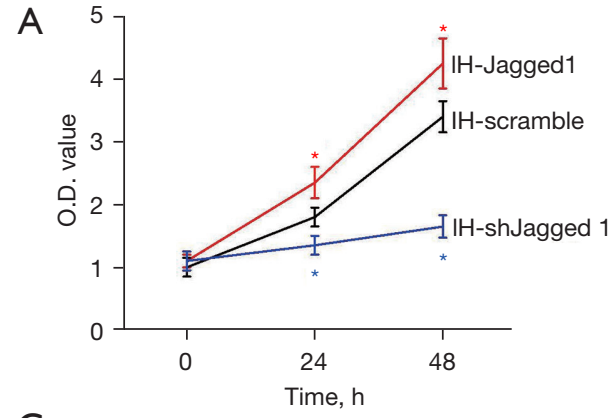

C

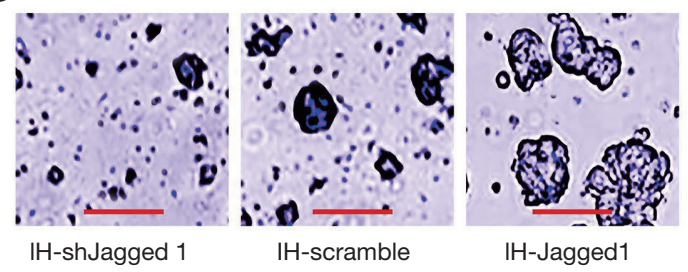

B

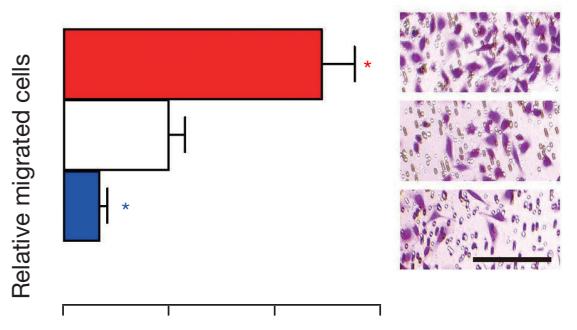

D

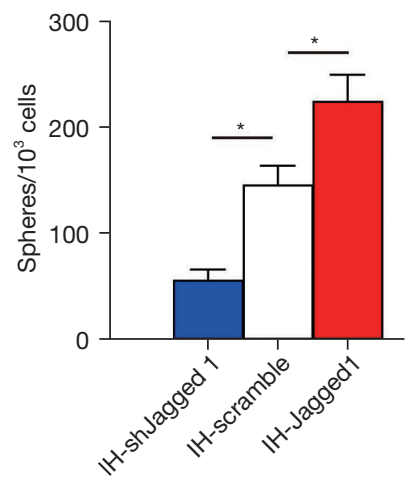

Figure 4 Altered Notch signaling and stemness of infantile hemangioma (IH) cells. (A) MTT assay for transduced IH cells. (B) Transwell cell migration assay of transduced IH cells. Red *: IH-Jagged1 versus IH-scramble. Blue *: IH-shJagged1 versus IH-scramble. Tumor sphere formation assay, shown by representative images $(C)$, and by quantification (D). $n=5 .{ }^{*} \mathrm{P}<0.05$. Scale bars $=50 \mu \mathrm{m}$.

Jagged1 significantly decreased the same protein levels in IH cells (Figure 3D,3E). Thus, this method successfully altered Notch signaling in IH.

\section{Effect of altered Notch signaling on stemness of IH cells}

Next, we examined the effects of Notch signaling on the stemness of IH cells in an MTT assay. Overexpression of Jagged1 significantly increased the growth of IH cells, while depletion of Jagged 1 significantly decreased the cells' growth (Figure 4A). Moreover, in the transwell cell migration assay, overexpression of Jagged 1 significantly increased the migratory potential of the IH cells, while depletion of Jagged1 significantly decreased it (Figure 4B). Furthermore, the golden standard for cancer cell stemness, namely tumor sphere formation, was assessed, showing that overexpression of Jagged 1 significantly increased sphere formation of IH cells, while depletion of Jagged1 significantly decreased it (Figure 4C,4D). Together, these data suggested that Notch signaling increased the stemness of IH cells.

\section{Effect of propranolol on the stemness of IH}

We have previously shown that propranolol dosedependently inhibits the growth of IH, but its effects on the activation of Notch signaling in IH are unknown. We addressed this question by firstly exposing IH cells to different doses of propranolol and measuring the levels of Jagged 1 and NICD in the treated IH cells. We found that $50 \mu \mathrm{mol} / \mathrm{L}$ propranolol had no effect, but $100 \mu \mathrm{mol} / \mathrm{L}$ significantly decreased the levels of Jagged 1 and NICD in IH cells, the degree of which was, however, not further increased when the dose of propranolol was increased to $150 \mu \mathrm{mol} / \mathrm{L}$ (Figure $5 \mathrm{~A}$ ). Moreover, the effects of propranolol on the protein levels of Jagged1, NICD and HES1 in IH cells confirmed the effects of propranolol on Jagged 1 and NICD mRNA (Figure 5B). These data were consistent with our previous research, which showed that the optimal dose of propranolol to suppress IH growth was $100 \mu \mathrm{mol} / \mathrm{L}$. In order to know whether the inhibitory effect of propranolol on IH growth is mediated by suppression of Notch signaling, we examined the rescue potential of overexpression of Jagged 1 in $100 \mu \mathrm{mol} / \mathrm{L}$ 

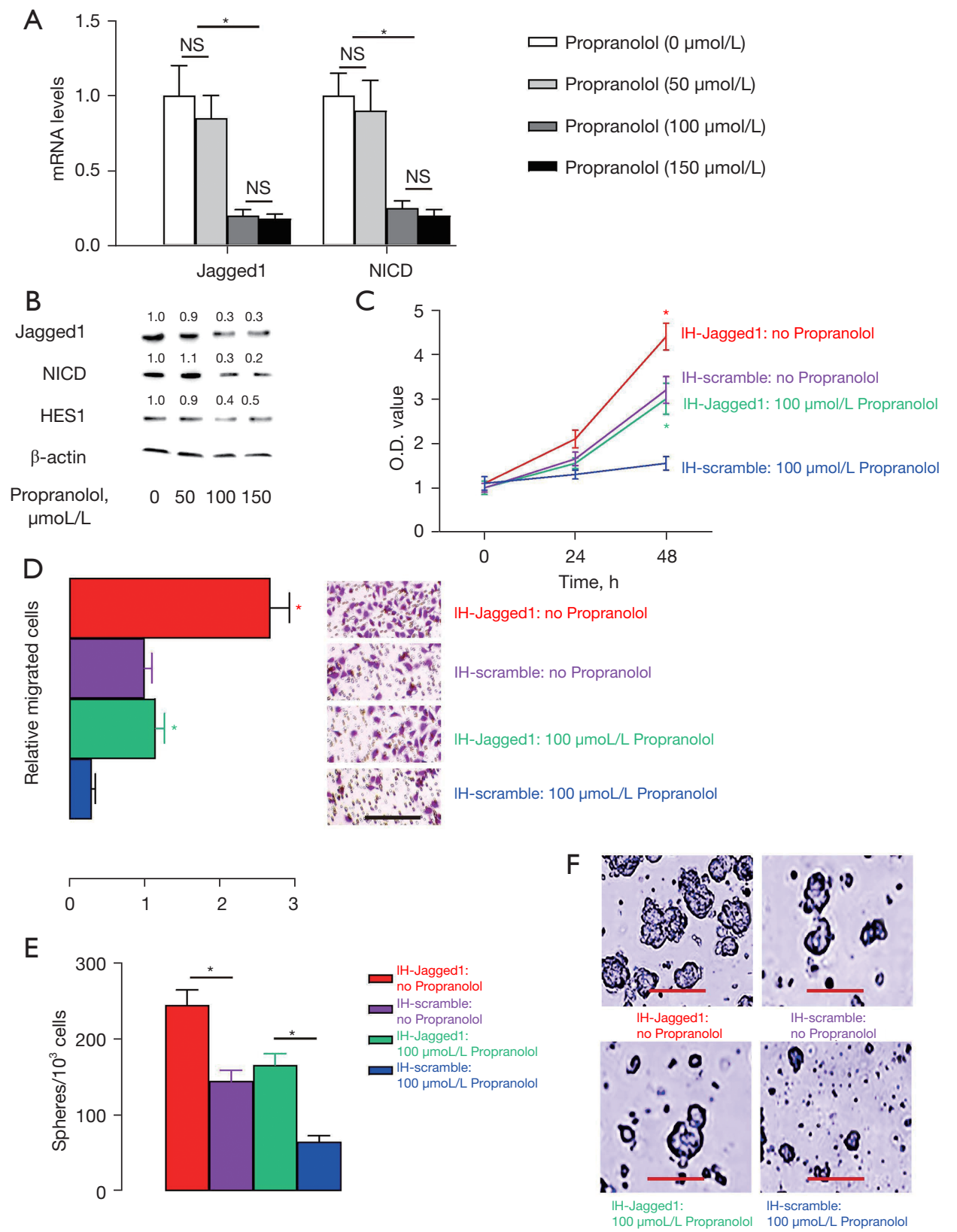

Figure 5 Effect of propranolol on stemness of infantile hemangioma (IH) cells. (A) IH cells exposed to different doses of propranolol and the levels of Jagged 1 and NICD in the treated IH cells were determined by RT-qPCR. (B) Western blot for Jagged1, NICD and HES1. (C) MTT assay under different conditions. (D) Transwell cell migration assay under different conditions. Tumor sphere formation assay for different conditions, shown by representative images $(\mathrm{E})$, and by quantification $(\mathrm{F}) . \mathrm{n}=5$. NS, non-significant. *P<0.05. Red *: IHJagged 1 versus IH-scramble that was exposed to null propranolol. Green *: IH-Jagged1 versus IH-scramble that was exposed to 100 momol/L propranolol. Scale bars $=50 \mu \mathrm{m}$. Notch intracellular domain. Hes1: hairy and enhancer of split-1.

propranolol-treated IH cells. For this, both IH-scrambled and IH-Jagged 1 cells were exposed to either $100 \mu \mathrm{mol} / \mathrm{L}$ propranolol or control solution. In the MTT assay, we found that overexpression of Jagged 1 abolished the inhibitory effect of $100 \mu \mathrm{mol} / \mathrm{L}$ propranolol on IH cell growth (Figure 5C). In the transwell cell migration assay, we found that overexpression of Jagged 1 abolished the inhibitory effects of $100 \mu \mathrm{mol} / \mathrm{L}$ propranolol on $\mathrm{IH}$ cell 

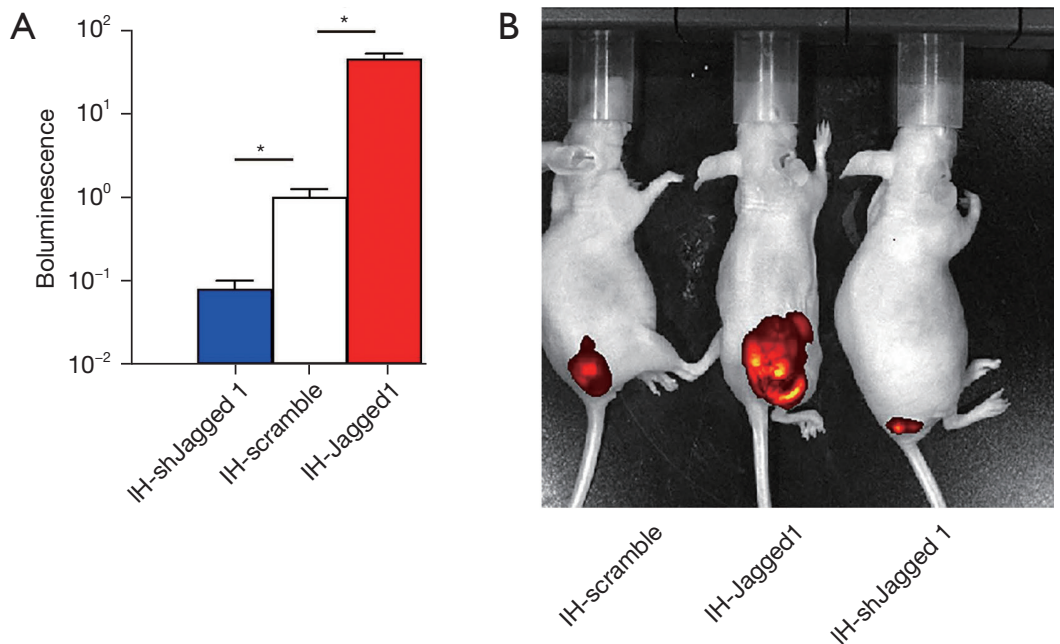

Figure 6 Effect of activated Notch signaling on in vivo growth of infantile hemangioma (IH). Equal numbers of IH-Jagged1, IH-shJagged1 and IH-scramble cells (200 cells/mouse) were subcutaneously transplanted immediately above the root of the tail of nude mice. One month after transplantation, tumor formation was assessed by bioluminescence detection after a 5 -min i.v. injection of luciferin, shown by quantification (A), and by representative images (B). $\mathrm{n}=6 .{ }^{*} \mathrm{P}<0.05$.

migration (Figure 5D). In the tumor sphere formation assay, we found that overexpression of Jagged 1 abolished the inhibitory effects of $100 \mu \mathrm{mol} / \mathrm{L}$ propranolol on $\mathrm{IH}$ sphere formation (Figure $5 E, 5 F$ ). Together, these data suggested that propranolol inhibited the stemness of IH through suppression of Notch signaling.

\section{Effect of activated Notch signaling on in vivo IH growth}

Finally, the same number of IH-Jagged1, IH-shJagged1 and IH-scrambled IH cells were subcutaneously transplanted into nude mice, and tumor formation was assessed by bioluminescence. Significantly larger tumors were observed in the mice grafted with IH-Jagged1, compared with IHscramble-grafted mice, shown by quantification (Figure $6 A$ ), and by representative images (Figure $6 B$ ). On the other hand, significantly smaller tumors were observed in mice grafted with IH-shJagged1, compared with the IHscramble-grafted mice, shown by quantification (Figure $6 A$ ), and by representative images (Figure 6B). Together, these findings suggested that activated Notch signaling promoted in vivo $\mathrm{IH}$ growth.

\section{Discussion}

The Notch signaling pathway includes a group of highly conserved molecules that play critical functions in a diversity of developmental and homeostatic processes, among which stemness is a very important one (23). Many different regulatory mechanisms are organized together to shape the exact activity of the Notch signaling pathway, to generate the precise output appropriate for the particular context (24). Past studies have strongly demonstrated the involvement of Notch signaling in controlling tumor stemness in different cancers (25). Because CSCs usually contribute to chemoresistance, it is crucial to target them to improve the efficacy of chemotherapy. IH seem to possess unique CSCs that express CD133 (3). Of note, current understanding of CSCs suggests that a single marker or a few combined markers may not be sufficient to fully define and characterize CSCs in certain tumors (26). Indeed, the recent technical improvement of next-generation sequencing may substantially further our understanding of CSCs in many different cancers (27), as currently such investigation is lacking for $\mathrm{IH}$.

In this study, we concurrently studied the effect of propranolol as chemotherapy for IH and the role of Notch/ Jagged1 signaling in the stemness of IH. The rationale of this connection was the characteristic of propranolol as a specific non-selective $\beta 2-\mathrm{AR}$ blocker that has been shown to inhibit demethylation adrenaline-induced Jagged 1 expression (20), and that Jagged 1 and Notch3 are important downstream signaling cascades of $\beta 2-A R(19)$.

First, we detected significant upregulation of Notch3 
and Jagged 1 in IH. We have previously shown a dosedependent suppression of IH growth by propranolol (28), so in this study we wanted to understand whether propranolol inhibits IH growth through Notch signaling and in addition, modulation of the stemness of IH. For this purpose, we used a genetic modification strategy to alter Notch signaling through Jagged1. The degree that the transduced IH cells were altered by Jagged1/shJagged1 did not exceed that in the primary IH specimens or in a published public database, suggesting that our experiment was clinically relevant.

The important finding of this study that propranolol inhibited the stemness of IH through suppressing of Notch signaling is a critical step for future improvement of chemotherapy for IH by targeting Notch signaling. It is noteworthy that we did not study the interplay of IH cells with other cell types inside the tumor (e.g., endothelial cells or mesenchymal cells). The interactions among these cells are known to greatly affect the tumorigenesis and homeostasis of IH, and Notch/Jagged1 signaling would be involved in not only the crosstalk between tumor cells, but also between tumor cells and non-tumor cells. Our study should inspire future studies of these aspects.

\section{Acknowledgments}

Funding: This work was supported by the National Natural Science Foundation Youth Science Fund Project (No. 81701949) and the Science and Technology Innovation Project of Shanghai Jiao Tong University School of Medicine (No. 16XJ21004).

\section{Footnote}

Reporting Checklist: The authors have completed the ARRIVE reporting checklist. Available at https://dx.doi. org/10.21037/atm-21-5563

Data Sharing Statement: Available at https://dx.doi. org/10.21037/atm-21-5563

Conflicts of Interest: All authors have completed the ICMJE uniform disclosure form (available at https://dx.doi. org/10.21037/atm-21-5563). The authors have no conflicts of interest to declare.

Ethical Statement: The authors are accountable for all aspects of the work in ensuring that questions related to the accuracy or integrity of any part of the work are appropriately investigated and resolved. Animal experiments were performed under a project license (No. XHEC-F-2021-072) granted by Ethics Committee of Shanghai Jiao Tong University School of Medicine. All animal experiments were carried out in compliance with the relevant guidelines and regulations for the care and use of animals issued by the Institutional Research Ethics Committee of Shanghai Jiao Tong University. A protocol was prepared before the study without registration. All procedures performed in this study involving human participants were in accordance with the Declaration of Helsinki (as revised in 2013). The study was approved by Institutional Ethics Board of the Shanghai Jiao Tong University School of Medicine (No. XHEC-D-2021-161) and informed consent was taken from all the patients.

Open Access Statement: This is an Open Access article distributed in accordance with the Creative Commons Attribution-NonCommercial-NoDerivs 4.0 International License (CC BY-NC-ND 4.0), which permits the noncommercial replication and distribution of the article with the strict proviso that no changes or edits are made and the original work is properly cited (including links to both the formal publication through the relevant DOI and the license). See: https://creativecommons.org/licenses/by-nc-nd/4.0/.

\section{References}

1. Rotter A, Samorano LP, de Oliveira Labinas GH, et al. Ultrasonography as an objective tool for assessment of infantile hemangioma treatment with propranolol. Int J Dermatol 2017;56:190-4.

2. Stiles J, Amaya C, Pham R, et al. Propranolol treatment of infantile hemangioma endothelial cells: A molecular analysis. Exp Ther Med 2012;4:594-604.

3. Kleinman ME, Tepper OM, Capla JM, et al. Increased circulating AC133+ CD34+ endothelial progenitor cells in children with hemangioma. Lymphat Res Biol 2003;1:301-7.

4. Wen L, Wang Y, Wen N, et al. Role of Endothelial Progenitor Cells in Maintaining Stemness and Enhancing Differentiation of Mesenchymal Stem Cells by Indirect Cell-Cell Interaction. Stem Cells Dev 2016;25:123-38.

5. Shah D, Wyatt D, Baker AT, et al. Inhibition of HER2 Increases JAGGED1-dependent Breast Cancer Stem Cells: Role for Membrane JAGGED1. Clin Cancer Res 2018;24:4566-78. 
6. Mancini SJ, Mantei N, Dumortier A, et al. Jagged1dependent Notch signaling is dispensable for hematopoietic stem cell self-renewal and differentiation. Blood 2005;105:2340-2.

7. Oktem G, Sanci M, Bilir A, et al. Cancer stem cell and embryonic development-associated molecules contribute to prognostic significance in ovarian cancer. Int J Gynecol Cancer 2012;22:23-9.

8. Labsch S, Liu L, Bauer N, et al. Sulforaphane and TRAIL induce a synergistic elimination of advanced prostate cancer stem-like cells. Int J Oncol 2014;44:1470-80.

9. Xiao W, Gao Z, Duan Y, et al. Notch signaling plays a crucial role in cancer stem-like cells maintaining stemness and mediating chemotaxis in renal cell carcinoma. J Exp Clin Cancer Res 2017;36:41.

10. Papadakos KS, Bartoschek M, Rodriguez C, et al. Cartilage Oligomeric Matrix Protein initiates cancer stem cells through activation of Jagged1-Notch3 signaling. Matrix Biol 2019;81:107-21.

11. Jiang H, Zhou C, Zhang Z, et al. Jagged1-Notch1deployed tumor perivascular niche promotes breast cancer stem cell phenotype through Zeb1. Nat Commun 2020;11:5129.

12. Nwabo Kamdje AH, Bassi G, Pacelli L, et al. Role of stromal cell-mediated Notch signaling in CLL resistance to chemotherapy. Blood Cancer J 2012;2:e73.

13. Liu S, Breit S, Danckwardt S, et al. Downregulation of Notch signaling by gamma-secretase inhibition can abrogate chemotherapy-induced apoptosis in T-ALL cell lines. Ann Hematol 2009;88:613-21.

14. Nefedova Y, Sullivan DM, Bolick SC, et al. Inhibition of Notch signaling induces apoptosis of myeloma cells and enhances sensitivity to chemotherapy. Blood 2008;111:2220-9.

15. Calicchio ML, Collins T, Kozakewich HP. Identification of signaling systems in proliferating and involuting phase infantile hemangiomas by genome-wide transcriptional profiling. Am J Pathol 2009;174:1638-49.

16. Wu JK, Kitajewski JK. A potential role for notch signaling in the pathogenesis and regulation of hemangiomas. J Craniofac Surg 2009;20 Suppl 1:698-702.

Cite this article as: $\mathrm{Ma} \mathrm{X}, \mathrm{Lv} \mathrm{K}, \mathrm{Wu} \mathrm{L}$, Ouyang $\mathrm{T}$. Propranolol inhibits stemness of hemangioma through Jagged1. Ann Transl Med 2021;9(22):1682. doi: 10.21037/atm-21-5563
17. Edwards AK, Glithero K, Grzesik P, et al. NOTCH3 regulates stem-to-mural cell differentiation in infantile hemangioma. JCI Insight 2017;2:93764.

18. Boscolo E, Stewart CL, Greenberger S, et al. JAGGED1 signaling regulates hemangioma stem cell-to-pericyte/ vascular smooth muscle cell differentiation. Arterioscler Thromb Vasc Biol 2011;31:2181-92.

19. Ferrari-Toninelli G, Bonini SA, Uberti D, et al. Notch activation induces neurite remodeling and functional modifications in SH-SY5Y neuronal cells. Dev Neurobiol 2009;69:378-91.

20. Rotter A, de Oliveira ZNP. Infantile hemangioma: pathogenesis and mechanisms of action of propranolol. J Dtsch Dermatol Ges 2017;15:1185-90.

21. Li HH, Lou Y, Zhang RR, et al. Propranolol Accelerats Hemangioma Stem Cell Transformation Into Adipocyte. Ann Plast Surg 2019;83:e5-e13.

22. Yeung ML, Houzet L, Yedavalli VS, et al. A genome-wide short hairpin RNA screening of jurkat T-cells for human proteins contributing to productive $\mathrm{HIV}-1$ replication. J Biol Chem 2009;284:19463-73.

23. Zieba JT, Chen YT, Lee BH, et al. Notch Signaling in Skeletal Development, Homeostasis and Pathogenesis. Biomolecules 2020;10:332.

24. Harper JA, Yuan JS, Tan JB, et al. Notch signaling in development and disease. Clin Genet 2003;64:461-72.

25. Alketbi A, Attoub S. Notch Signaling in Cancer: Rationale and Strategies for Targeting. Curr Cancer Drug Targets 2015;15:364-74.

26. Brown DV, Mantamadiotis T. Insights into the next generation of cancer stem cell research. Front Biosci (Landmark Ed) 2014;19:1015-27.

27. Mardis ER. The Impact of Next-Generation Sequencing on Cancer Genomics: From Discovery to Clinic. Cold Spring Harb Perspect Med 2019;9:a036269.

28. Ma X, Zhao T, Ouyang T, et al. Propranolol enhanced adipogenesis instead of induction of apoptosis of hemangiomas stem cells. Int J Clin Exp Pathol 2014;7:3809-17.

(English Language Editor: K. Brown) 
Supplementary

Table S1 Summary of patients' characteristics and diagnosis details

\begin{tabular}{lccccc}
\hline Patient number & Gender & Age of surgery (months) & Infantile hemangioma $(\mathrm{IH})$ position & Diagnosis & IH on other sites \\
\hline 1 & M & 6.7 & Perioral & MRI, B ultrasound & No \\
2 & F & 5.4 & Perioral & MRI & MRI \\
3 & F & 8.5 & Nose & MRI, B ultrasound & No \\
4 & F & 9.2 & Perioral & MRI & No \\
5 & M & 4.3 & & rris
\end{tabular}

